\title{
Analysis of Efficiently Lossless Compression of Color Image by using Deferent Algorithms
}

\author{
Siddharth Sinha \\ Dept. of Electronics and Communication Engg. \\ Lakshmi Narain College of Technology and Science \\ Bhopal, India
}

\author{
Ayoush Johari \\ Dept. of Electronics and Communication Engg. \\ Lakshmi Narain College of Technology and Science \\ Bhopal, India
}

\begin{abstract}
Digital Color Image required large amount of space to store and large bandwidth to transmit it. Due to limitations in bandwidth and in storage space, it is vital requirement to compresses digital color image. Color image compression is required with negligible loss in image quality for the effective use of further restored image. To meet this, numerous image compression techniques are developed in last several years. This paper analyses these techniques and presents a compression among them to find out the best method for digital color image compression. The objective of this paper is also to find out the best approach for developing new way of digital color image compression with minimum loss in image quality.
\end{abstract}

\section{General Terms}

ECG, ECG De-noising, Baseline Wader Noise, PSD, SNR

\section{Keywords}

Electrocardiogram, Baseline Wander Noise, FIR Filter, IIR Filter, Independent Component Analysis.

\section{INTRODUCTION}

The approach of high velocity registering gadgets and quick improvement in the field of communication has made a huge open door for different machine based image applications. The measure of information needed to store a digital image is ceaselessly expanding and overpowering the stockpiling gadgets. Image compression is putting away images utilizing lesser number of bits than its unique size. Increment in image related application has made an issue of image putting away and transmission. Putting away and transmission of images oblige impressive measure of space and bandwidth. Image compression addresses this issue. It lessens the quantity of bits needed to speak to the image. Subsequently, in the period of digital communication, image compression is vital field of examination [1]. There are two sorts of compression techniques: Lossless compression and Lossy compression.

In lossless compression, reconstructed image is precisely same as compressed image i.e if information have been losslessly compressed, the first information can be recouped precisely from the compressed information. It is by and large utilized for applications that can't permit any contrast between the first and reconstructed information [2].

In lossy compression high compression ratio is accomplished at the expense of a few blunders in reconstructed image. It is dependably not important to get careful image after recreation. Image with great perceptual quality with some blunder is worthy in a few applications. For instance, it is worthy that a reconstructed feature sign is not the same as the first the length of the distinctions don't bring about irritating antiquities. Then again, we can for the most part acquire higher compression ratios than is conceivable with lossless compression. With stripped eyes, viewer can't recognize the contrast between unique image and decompressed image. Consequently commonly lossy compression is favored over lossless compression. Most generally utilized lossy compression strategy is transform coding, for example, Discrete Cosine Transform (DCT) utilized as a part of JPEG and Wavelet transform utilized as a part of Jpeg2000. Compression ratio and image nature of decompressed image, these are two noteworthy things to be considered in image compression. As compression ratio expands, nature of reconstructed image begins corrupting. Numerous compression methods like Vector Quantization (VQ), Predictive Coding (PC), Transform Coding (TC), Differential Image Coding (DIC) have been presented [3].

In transform coding, at first DCT was prevalent image compression method. DCT shows straightforwardness and acceptable execution in compression. As it is connected on blocked image relationship over the piece limits can't be killed. It presents blocking antiques particularly at low bit rate. This downside was overcome by wavelet transform. Since last two to three decades wavelets have come into picture and turned into an appealing strategy for image compression. It gives time and frequency examination of information. Wavelet transform can be specifically connected to entire image without blocking it. Wavelet based coding is more powerful under transmission and deciphering slips. Multiresolution property of wavelet transforms help to view the image at distinctive scales. Late pattern is to utilize hybrid strategy for image compression. In hybrid method, one transform is consolidated with an alternate transform to incorporate the favorable circumstances of both transforms. At times mix of VQ and transform additionally has been utilized [4]-[7].

\section{IMAGE COMPRESSION TECHNIQUES CLASSIFICATION}

Image coder framework contains three associated parts specifically source encoder, quantizer and entropy encoder. Image compression comprises of applying a transform to decorrelate the source information, for example, image or feature, quantizing the transform coefficients lastly changing over the quantized qualities to entropy in Fig. 1. Taking into account processing calculations, compression methods are separated into two noteworthy classifications, for example, misfortune / lossless based compression and predication / transform based compression. 


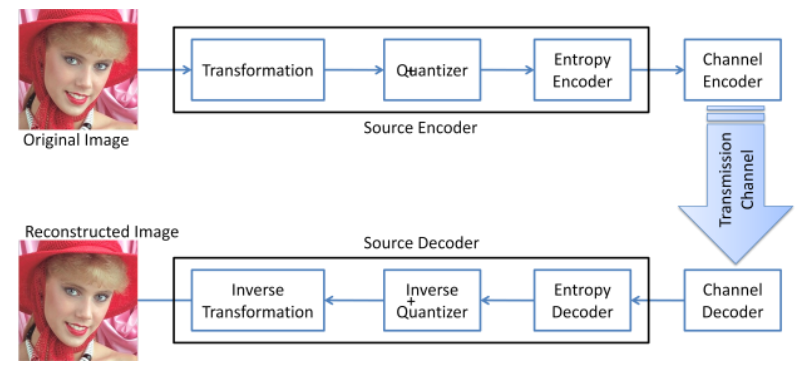

Fig.1 Typical Image Transmission System

\subsection{Lossless Compression}

Lossless compression is numerically indistinguishable to the first image. It is overall called as silent compression. It is characterized into Huffman coding, Arithmetic decomposition, Lempel zin and Run Length Encoding (RLE) .

\subsection{Lossy Compression}

Calculations that restore the presentation to be like the first image are called lossy systems. Recreation of image is a close estimation of unique image, so the need of estimation of nature of the image for lossy compression strategy. It gives high compression ratio and frequently connected in feature and image compression than lossless systems. It is characterized into frequency-arranged procedures, expectation based methods and imperativeness situated strategies. Data officially sent to anticipate resulting values by watching past qualities, for example, movement remuneration, Adaptive digital pulse code modulation is called prescient Based strategies. The image is transformed to spatial area or frequency space, for example, Discrete Cosine Transform (DCT), sub band decomposition, wavelet transforms and different transforms, which gives more prominent information compression at the cost of more noteworthy processing is called Frequency arranged procedures. The premise for compression utilizes different qualities of images, for example, sifting for instance DVI strategy uses to color up information separating and lookup tables, sub examining, bit assignment, quantization, for example, scalar and vector are called essentialness arranged methods[9]-[10].

\subsection{Hybrid Compression Techniques}

JPEG, MPEG and Kohonen's Self Organizing Feature Map (SOFM), Set Partitioning in Hierarchical Trees (SPIHT) combine various approaches such as Vector Quantization (VQ) compression and DCT based compression or Differential Pulse Code Modulation (DPCM).

\section{IMAGE COMPRESSION TECHNIQUES}

\subsection{Discrete Cosine Transform based Compression}

DCT is a Fourier-related transform like the discrete Fourier transform (DFT), however utilizing just genuine numbers. Dcts are proportionate to Dfts of generally double the length, working on genuine information with even symmetry (since the Fourier transform of a genuine and even capacity is genuine and even), where in a few variations the data and/or yield information are moved considerably a specimen. There are eight standard DCT variations, of which four are regular. The most widely recognized variation of discrete cosine transform is the sort II DCT, which is frequently called essentially "the Dct",[1]-[2] its converse, the sort III DCT, is correspondingly regularly called basically "the reverse DCT" or "the IDCT". Two related transforms are the discrete sine transform (DST), which is equal to a DFT of genuine and odd capacities, and the altered discrete cosine transform (MDCT), which is focused around a DCT of covering information.

The DCT is utilized as a part of JPEG image compression, MJPEG, MPEG, DV, Daala, and Theora feature compression. There, the two-dimensional DCT-II of $\mathrm{N} \mathrm{X} \mathrm{N} \mathrm{squares} \mathrm{are}$ registered and the results are quantized and entropy coded. For this situation, $\mathrm{N}$ is commonly 8 and the DCT-II equation is connected to each one column and section of the piece. The result is a $8 \times 8$ transform coefficient show in which the $(0,0)$ component (upper left) is the DC (zero-frequency) part and sections with expanding vertical and level record qualities speak to higher vertical and flat spatial frequencies [3].

\subsection{Reversible Integer Discrete Cosine Transforms (RDCT)}

Image compression is accomplished utilizing RDCT. It is gotten from the network factorization hypothesis. Existing Techniques focused around the discrete cosine transform to experience the ill effects of poor execution when contrasted and wavelet image codec in lossy image compression. RDCT joined with different dynamic codec, for example, SPHIT and SPECK. The coefficients are revamped into sub-band structure and coded by setting based piece coding. The DWT based SPHIT supplanted by RDCT with channels. It gives elite in both lossless/lossy compression and utilized for square source pixels good for piece transform by prefilter and utilized for decreasing piece relics by post channel [4].

\subsection{Integer DCT and I2IDCT}

In lossless compression, the rough guess of DCT matrix, the low cost 8-point number DCT (IntDCT) is focused around Walsh Hadamard Transform (WHT) and number lifting. I2idet: The $\mathrm{N}$ point I2I-DCT is accomplished by applying recursive systems and lifting procedures where $\mathrm{N}$ is in force of two for lossless compression and this is proposed by abhayaratne [5]-[7].

\subsection{Fractal Compression}

Fractal compression is a lossy compression system for digital images, in light of fractals. The strategy is best suited for compositions and common images, depending on the way that parts of an image regularly take after different parts of the same image. Fractal calculations change over these parts into scientific information called "fractal codes" which are utilized to reproduce the encoded image.

It is great scientific encoding casing. The premise for this compression is the estimate of unique image focused around arrangement hypothesis and settled point hypothesis for the development of a nearby Iterated Function System (IFS). Diverse strategies have considered for parallel encoding phase of this compression, for example, granularity, information parcel and burden adjusting [8]. Fractal Methodology uses determination. The compression ratio is more prominent than $16 \%$ in 1992 and the drawback is the slow encoding system.

\subsection{EZDCT and TDCT}

Discrete cosine based installed image coder is called Embedded Zero Discrete Cosine Transform (EZDCT) for lossy compression. Tran et al. outline Time Domain Lapped Transform (TDLT) and it is attained by adding preand post channel to the DCT. 


\subsection{Discrete Wavelet Transform based Compression}

Frequently flags we wish to process are in the time-area, however so as to process them all the more effortlessly other data, for example, frequency is needed. Numerical transforms interpret the data of signs into distinctive representations. For instance, the Fourier transform changes over a sign between the time and frequency spaces, such that the frequencies of a sign can be seen. However the Fourier transform can't give data on which frequencies happen at particular times in the sign as time and frequency are seen freely. To take care of this issue the Short Term Fourier Transform (STFT) presented the thought of windows through which distinctive parts of a sign are seen. For a given window in time the frequencies can be seen. However Heisenburg's Uncertainty Principle expresses that as the determination of the sign enhances in the time space, by zooming on diverse segments, the frequency determination deteriorates. Conceivably, a technique for multiresolution is required, which allows certain parts of the sign to be determined well in time, and different parts to be determined well in frequency [11]. The force and enchantment of wavelet investigation is precisely this multiresolution. Images contain a lot of data that obliges much storage room, huge transmission bandwidths and long transmission times. Hence it is beneficial to pack the image by putting away just the fundamental data expected to reproduce the image. An image can be considered a matrix of pixel (or power) values. Keeping in mind the end goal to clamp the image, redundancies must be abused, for regions where there is almost no change between pixel values. Consequently images having vast zones of uniform shade will have substantial redundancies, and on the other hand images that have successive and huge changes in color will be less excess and harder to pack [12].

Wavelet examination can be utilized to separation the data of an image into estimate and subtle element and subsignal. Subsignal demonstrates the general pattern of pixel qualities, and three subtle element subsignals demonstrate the vertical, even and slanting points of interest or changes in the image. In the event that these points of interest are little then they can be set to zero without altogether changing the image. The worth below which subtle elements are viewed as sufficiently little to be set to zero is known as the edge. The more prominent the quantity of zeros the more noteworthy the compression that can be attained. The measure of data held by an image after compression and decompression is known as the vitality held and this is corresponding to the total of the squares of the pixel values. On the off chance that the vitality held is $100 \%$ then the compression is known as lossless, as the image can be reconstructed precisely. This happens when the edge worth is situated to zero, implying that the subtle element has not been changed. On the off chance that any qualities are changed then vitality will be lost and this is known as lossy compression. Conceivably, amid compression the quantity of zeros and the vitality maintenance will be as high as could be expected under the circumstances. Nonetheless, as more zeros are gotten more vitality is lost, so a harmony between the two needs to be found.

\subsection{VQ Compression}

Vector Quantization is the most ideal method for quantizing and layering the images. The central thought is to secure a codebook comprising of code vectors such that each one code vector can speak to a set of image pieces of size $m \times m$. An image is first segment into $m \times m$ non-covering square, which is spoken to as m2-tuple vectors are called preparing vectors.
Vector Quantization (VQ) gives preferable execution over Scalar Quantization (SQ). SAR image work better for VQ compression since quantization is first approach for SAR images. The essential work is to plan an adaptable Codebook and Nasrabadi and King gives a decent survey of VQ. Chen's correlation shows that a codebook created focused around (Linde, Buzo and Gray) LBG calculation has higher PSNR values over some different plans slow disconnected from the net preparing [14]. It is the straightforward decoder and preferences are no coefficient quantization and not proper for low bit rate compression, the measure of reckonings amid the quest for ideal code vector in encoding little bits every pixel furthermore slow codebook generation or slow disconnected from the net preparing. The compression ratio is short of what $32 \%$.

\subsection{Hybrid Wavelet Transforms}

The idea of producing hybrid wavelet transform from two orthogonal transforms and amplifies it with diverse orthogonal transform like DCT, WALSH, DHT, DKT and so forth. Creating wavelet transform from orthogonal transform frameworks of two separate sizes from two orthogonal transform networks $\mathrm{A}_{\mathrm{M}}$ of size MxM as indicated in Fig.2 and $\mathrm{B}_{\mathrm{N}}$ of size $\mathrm{NxN}$ as demonstrated in Fig.3, we can generate wavelet transform matrix $\mathrm{T}$ of size MNxMN. Case in point, from orthogonal transform matrix $A_{M}$ of size $32 \times 32$ and $B$ of size $8 \times 8$, we can generate wavelet transform matrix of size $256 \times 256$. To generate first $\mathrm{N}$ lines of wavelet transform matrix, duplicate every segment of matrix B with every coefficient of the first line of matrix A. To generate next $\mathrm{N}$ lines, second column of $A_{M}$ is affixed with zeros and afterward it is movement pivoted [15]. Comparable methodology is rehashed for remaining columns of A from third column onwards. This whole strategy is demonstrated in Fig.4

$\mathbf{A}_{\mathbf{M}}=$\begin{tabular}{|c|c|c|c|}
\hline $\mathrm{A}_{11}$ & $\mathrm{~A}_{12}$ & $\ldots \ldots$ & $\mathrm{A}_{1 \mathrm{M}}$ \\
\hline $\mathrm{A}_{21}$ & $\mathrm{~A}_{22}$ & $\ldots \ldots$ & $\mathrm{A}_{2 \mathrm{M}}$ \\
\hline$:$ & $:$ & $\ldots \ldots$ & $:$ \\
\hline $\mathrm{A}_{\mathrm{M} 1}$ & $\mathrm{~A}_{\mathrm{M} 2}$ & $\ldots \ldots$ & $\mathrm{A}_{\mathrm{MM}}$ \\
\hline
\end{tabular}

Fig.2. MXM Orthogonal Transform Matrix A

$\mathbf{B}_{\mathbf{N}}=$\begin{tabular}{|c|c|c|c|}
\hline $\mathrm{B}_{11}$ & $\mathrm{~B}_{12}$ & $\ldots \ldots$ & $\mathrm{B}_{1 \mathrm{~N}}$ \\
\hline $\mathrm{B}_{21}$ & $\mathrm{~B}_{22}$ & $\ldots \ldots$ & $\mathrm{B}_{2 \mathrm{~N}}$ \\
\hline$:$ & $:$ & $\ldots \ldots$ & $:$ \\
\hline $\mathrm{B}_{\mathrm{N} 1}$ & $\mathrm{~B}_{\mathrm{N} 2}$ & $\ldots \ldots$ & $\mathrm{B}_{\mathrm{NN}}$ \\
\hline
\end{tabular}

Fig.3. NXN Orthogonal Transform Matrix B

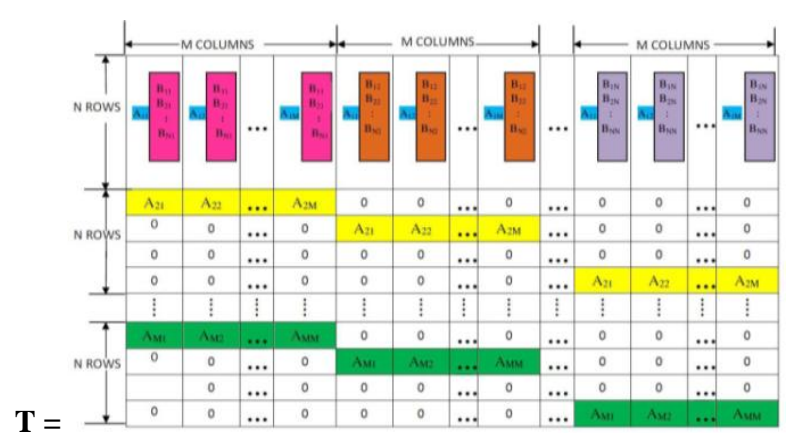

Fig.4. Generation of Wavelet Transform $\mathrm{T}$ from Orthogonal Transforms A and B 


\section{IMAGE COMPRESSION EVALUATION PARAMETERS 4.1 Mean Square Error (MSE)}

The MSE is the total squared error between the compressed and the first image. A lower estimation of MSE means lesser error, and it has the opposite connection with PSNR. Mean square error is a paradigm for an estimator: the decision is the particular case that minimizes the aggregate of squared errors because of inclination and because of change. As a rule, it is the normal of the square of the distinction between the craved reaction and the genuine framework yield. As a misfortune capacity MSE is likewise called squared error misfortune. MSE measures the normal of the square of the 'error'. The MSE is the second snippet of the error, and accordingly incorporates both the change of the estimator and its predisposition. For an unprejudiced estimator, the MSE is the change. In a similarity to standard deviation, taking the square foundation of MSE yields the root mean squared error or RMSE. For a fair-minded estimator, the RMSE is the square foundation of the fluctuation, known as the standard error.

$$
M S E=\frac{1}{m X n} \sum_{y=1}^{m} \sum_{x=1}^{n}\left[I(x, y)-I^{\prime}(x, y)\right]^{2}
$$

Where, $I(x, y)$ is the original image and $I^{\prime}(x, y)$ is the reconstructed image and $\mathrm{m}, \mathrm{n}$ are the dimensions of the image. Lower the value of MSE, the lower the error and better picture quality.

\subsection{Peak Signal to Noise Ratio (PSNR)}

PSNR is a measure of the top error. Numerous signs have wide dynamic reach, in view of that reason PSNR is generally communicated as far as the logarithmic decibel scale in $(\mathrm{db})$. Typically, a higher estimation of PSNR is great in light of the fact that it implies that the ratio of sign to commotion is higher. Here, a sign speaks to unique image and commotion speaks to the error in recreation. It is the ratio between the greatest conceivable force of a sign and the force of the adulterating commotion. PSNR diminishes as the compression ratio increments for an image. The PSNR is characterized as:

$$
P S N R=10 * \log _{10}\left\{\frac{M A X I^{2}}{M S E}\right\}=20 * \log _{10}\left\{\frac{M A X I}{\sqrt{M S E}}\right\}
$$

\subsection{Compression ratio $\left(C_{R}\right)$}

Compression ratio (CR) is a measure of the decrease of the itemized coefficient of the information. At the present time image compression, it is essential to know what amount definite (vital) coefficient one can toss from the info information to haven basic data of the first information. Compression ratio can be communicated as:

$$
C_{R}=\frac{\text { Decompresed Image }}{\text { Orignal Image }}
$$

\subsection{Subjective Evaluation Parameter}

The visual impression of the reconstructed image is fundamental. Sometimes the target quality appraisal does not give legitimate data about the nature of the reconstructed image. In such situations, it is vital to examine the reconstructed image utilizing subjective investigation that implies by human perceptual framework. At the point when the subjective measure is considered, viewers concentrate on the contrast in the middle of reconstructed and unique image and associates the distinctions.

\section{CONCLUSIONS}

The study summarizes the need for compression and classification of compression techniques. A survey is performed on the most essential and advance compression methods, including coding techniques based on DCT, DWT, VQ, Fractal approach and other methods. The compression between Wavelet, JPEG, VQ and Fractal approach is also shown in Table 1. All these studies show that the wavelet approach seems much better than other approaches. The hybrid wavelet approach by using DCT, DKT and DHT is another best approach for efficient lossles compression.

\section{ACKNOWLEDGMENT}

The author would like to express their sincere thanks to Dr. Soni Changlani, Head of the Department of Electronics and Communication, all authors the reference for this research, All faculty members of the department and of all the persons who have supported in any way for this survey.

\begin{tabular}{|c|c|c|c|c|}
\hline Method & Advantages & Disadvantages & $\begin{array}{c}\text { Average } \\
\text { PSNR } \\
\text { (dB) }\end{array}$ & $\begin{array}{c}\text { Compression } \\
\text { Ratio }\end{array}$ \\
\hline Wavelet & $\begin{array}{l}\text { High } \\
\text { Compression } \\
\text { Ratio }\end{array}$ & $\begin{array}{l}\text { Coefficient } \\
\text { Quantization Bit } \\
\text { allocation }\end{array}$ & 36.71 & >>32 \\
\hline JPEG & $\begin{array}{l}\text { Current } \\
\text { Standard }\end{array}$ & $\begin{array}{l}\text { Coefficient(DCT) } \\
\text { quantization } \\
\text { Bit allocation }\end{array}$ & 32.24 & $<=50$ \\
\hline VQ & $\begin{array}{l}\text { Simple } \\
\text { decoder } \\
\text { No- } \\
\text { coefficient } \\
\text { quantization }\end{array}$ & $\begin{array}{l}\text { Slow codebook } \\
\text { generation Small } \\
\text { bpp }\end{array}$ & 28.26 & $<32$ \\
\hline Fractal & $\begin{array}{l}\text { Good } \\
\text { mathematical } \\
\text { Encoding- } \\
\text { frame }\end{array}$ & Slow Encoding & 27.21 & $>=16$ \\
\hline
\end{tabular}

Table 1. Comparison between various method for Image Compression

\section{REFERENCES}

[1] Kumar, S., and Sood, V., Quality Assessment of colour image compression using Haar Wavelet Transform, International Journal of Engineering Trends and Technology, 266-269, 2012

[2] Kekre, H.B., Sarode, T., and Natu, P., Image Compression Using Real Fourier Transform, Its wavelet Transform and Hybrid Wavelet Transform, International Journal of Advanced Computer science and Application, 2013.

[3] Sunil, Kumar, V.V., and Reddy, M.I.S, Image compression technique by using wavelet transform, journal of Information Engineering and applications, 2012.

[4] Nadenau, M.J., Reichel, J., and Kunt, M., Wavelet Based Colour Image Compression: Exploiting the Contrast Sensitivity Function, IEEE Transactions Image Processing.

[5] Hekre, H.B., Sudeep, D., Thepade, and Parkar, A., A Comparison of Haar Wavelets and Kekree"s Wavelets for 
Storing Colour Informationin a Greyscale Image, International Journal of Computer Applications (IJCA),32-38, 2010.

[6] Hekre, H.B., Sudeep, D., Thepade, and Maloo, A., Performance Comparison of Image Retrieval Techniques using Wavelet Pyramids ofWalsh, Haar and Kekre Transforms, International Journal of Computer Applications (IJCA), 1-8, 2010.

[7] Hekre, H.B., Sudeep, D., Thepade, and Maloo, A., Face Recognition using Texture Features Extracted form Walshlet Pyramid, ACEEE International Journal on Recent Trends in Engineering and Technology (IJRTET), 2010

[8] Hekre, H.B., Sudeep, D., Thepade, and Maloo, A., Face Recognition using Texture Features Extracted from Haarlet Pyramid, International Journal of Computer Applications (IJCA), 41-45, 2010.

[9] Hekre, H.B., Sudeep, D., Thepade, and Natu, P., Image Compression based on Hybrid wavelet transform generated using orthogonal component transform of different sizes, International Journal of soft computing and Engineering, 2013.
[10] Walsh, J.L., A Closed Set of Orthogonal Functions, AmericanJournal of Mathematics, 5-24, 1923.

[11] Ahmed, N., Natarajan, T., and Rao, K. R., Discrete CosineTransform, IEEE Transaction Computers, 90-93, 1974.

[12] Chen, W., Smith, C. H., and Fralick, S. C., A Fast Computational Algorithm For The Discrete Cosine Transform, IEEE Transaction Communications, 10041008, 1977.

[13] Kekre, H.B., and Thepade, S.D., Image Retrieval using Non-Involutional Orthogonal Kekre's Transform, International Journal of Multidisciplinary Research and Advances in Engineering (IJMRAE), 189-203, 2009.

[14] Kekre, H.B., and Thepade, S.D., Athawale, A., Anant S., Prathamesh V., and Suraj S., Kekre Transform over Row Mean, Column Mean and Both using Image Tiling for Image Retrieval, International Journal of Computer and Electrical Engineering (IJCEE), 964-971, 2010.

[15] Hartley, R. V. L., A more symmetrical Fourier analysis applied to transmission problems, Proceedings of IRE 30, 144-150, 1942 